\title{
裂解气相色谱法快速鉴定斑点热群立 克次体的初步探讨
}

\author{
周方吕正文
}

(军事医学科学院微生物流行病研究所, 北京)

\section{一、引 言}

立克次体的常规检验方法包括分离培养、免疫血清检查. 此外, 还有组织培养、免疫茧光 染色和血凝试验等. 从病原体的分离培养到鉴定所需时间快则一月, 慢则数月,给临床诊断和 病原体的分类鉴定造成很大困难。因此，建立一种新的快速、敏感、特异、安全、简便的检验方 法是非常必要的.

本文报告用裂解与相色谱法 (Pyrolysis Gas Chromatography) 对来自世界卫生组织的 4 种 斑点热立克次体和我国新疆地区分离的精河株立克次体鸡肧卵黄襄材料的初步鉴别结果，并 比较了 4 种不同的裂解色谱实验条件. 看到了不同感染材料和正常鸡肧卵黄囊材料裂解气相 色谱图的差别，也看到了不同感染材料裂解图谱之间的差异. 从而揭示了裂解气相色谱法快 速鉴别斑点热群立克次体的可行性.

\section{二、材料和方法}

1. 实验毒种 自世界卫生组织引进的 4 种是: 立氏立克次体（Rickettsia rickettsii） $R$ 株, 小株立克次体（Rickettsia akari）Kaplan 株,派氏立克次体（Rickettsia parkeri）FS110 株和 蒙塔那立克次体（Rickettsia montana）B 株; 精河株立克次体系于 1977 年自我国新疆纳氏革蜱 (Dermacentor nuttalli) 分离.

2. 裂解气相色谱样品的制备 取上述各种立克次体 $20 \%$ 卵黄㐮悬液接种于 5 日砱鸡 肧, 每只 $0.5 \mathrm{ml}$, 于 $33^{\circ} \mathrm{C}$ 㽟育. 大多数鸡肧于接种后 $4-5$ 日死亡, 将已死鸡胚继续睬育 48 小 时, 取立克次体繁殖达甘一州之卵黄襄 2 个, 于 $120^{\circ} \mathrm{C}$ 干烤 4-6 小时, 研成细粉, 装瓶贮放干 燥器内备用.

按同样方法同时制备供正常对照用的鸡胚卵黄囊样品.

\section{3. 裂解气相色谱仪器系统及实验条件}

(1) 第一组: 上海分析仪器厂 100 型气相色谱仪和 SWK-4C 型数字温度程序控制仪. 居里点裂解器, 样品裂解温度 $770^{\circ} \mathrm{C}$, 裂解时间 12.5 尓. 氢焰电离鉴定器. 不锈钢色谱柱长 $2 \mathrm{~m}$, 内径 $4 \mathrm{~mm}$, 填充物为 Porapak Q. 将欲测各种干燥样品分别制成水悬液, 粘附于裂解丝 上,干燥. 详细方法见参考文献 [1].

（2）第二组：Hewlett-Packard 公司 $5840 \mathrm{~A}$ 型气相色谱仪, 双色谱柱, 双氢焰电离鉴定

本文 1982 年 11 月 11 日收到. 
器. CDS100 型固体样品裂解器. 选用白金丝螺旋形裂解探针, 裂解温度 $850^{\circ} \mathrm{C}$, 裂解时间 10 秒. 不锈钢色谱柱长 8 英尺, 内径 $1 / 8$ 英寸, 填充物为 Porapak Q. 程序升温色谱法. 将干燥 样品装入玻璃毛细管内, 称重后插入探针的白金丝螺旋内. 每次用样量为 80-100 微克. 柱 炉初始温度 $80^{\circ} \mathrm{C}, 1$ 分钟后以 $6^{\circ} \mathrm{C} /$ 分升至 $200^{\circ} \mathrm{C}$. 详细方法见参考文献 $[2,3]$.

(3) 第三组: 在这里以氮磷鉴定器取代氢焰电离鉴定器, 每次分析称取干燥样品量仅为 15-25 微克. 其他仪器及实验条件和第二组相同. 详细方法见参考文献 [4].

（4）第四组: 在这里用涂渍 FFAP 的上试 101 白色担体代替了 Porapak Q. 柱炉程序 升温初始温度为 $60^{\circ} \mathrm{C}, 1$ 分钟后以 $8^{\circ} \mathrm{C} /$ 分速率升至 $220^{\circ} \mathrm{C}$. 每次分析取样 $150-200$ 微克. 其 他仪器及实验条件和第二组相同.

\section{三、结 果}

在所选 4 组实验条件下, 分别绘制出正常对照和 5 种斑点热群立克次体的裂解气相色谱 图. 通过对这些化学指纹图的目测看到了正常对腭和感染斑点热立克次体鸡泼卵黄霊培养材 料之间的差异，以及各种感染材料图谱间的区别.

（1）由第一组条件得到的裂解图谱中只能看到正常对照和感染卵黄噻材料(图 1) 之间的 差别,图中阴影部分表示显著差异. 而 5 种感染材料的裂解图谱未见任何差异.

（2）由第二组条件绘制的裂解图谱除显示了正常对照和感染立克次体卵黄震材料的差别 外, 感染材料的图谱也有差别. 例如, 正常卵黄襄图谱中 $13,14,15$ 和 16 分钟处的色谱峰以 14 分钟处最高, 而感染材料图谱中则以 13 分钟处最高; 正常材料的 19 和 20 分钟处出现两个 色谱峰, 而感染材料则出现 3 个峰. 派氏 FS110 株 (图 2) 的 22 分钟处色谱峰比 23 分钟处 高, 而小株 Kaplan 株相应两个色谱峰则相反. 精河株和派氏 FS110 株很相似. 总的情况是, 这组感染材料图谱间的差别不如第三组条件所得的差别明显.

（3）第三组条件绘制的裂解图谱不但能显示出正常对照和感染材料图谱间的本质差别,

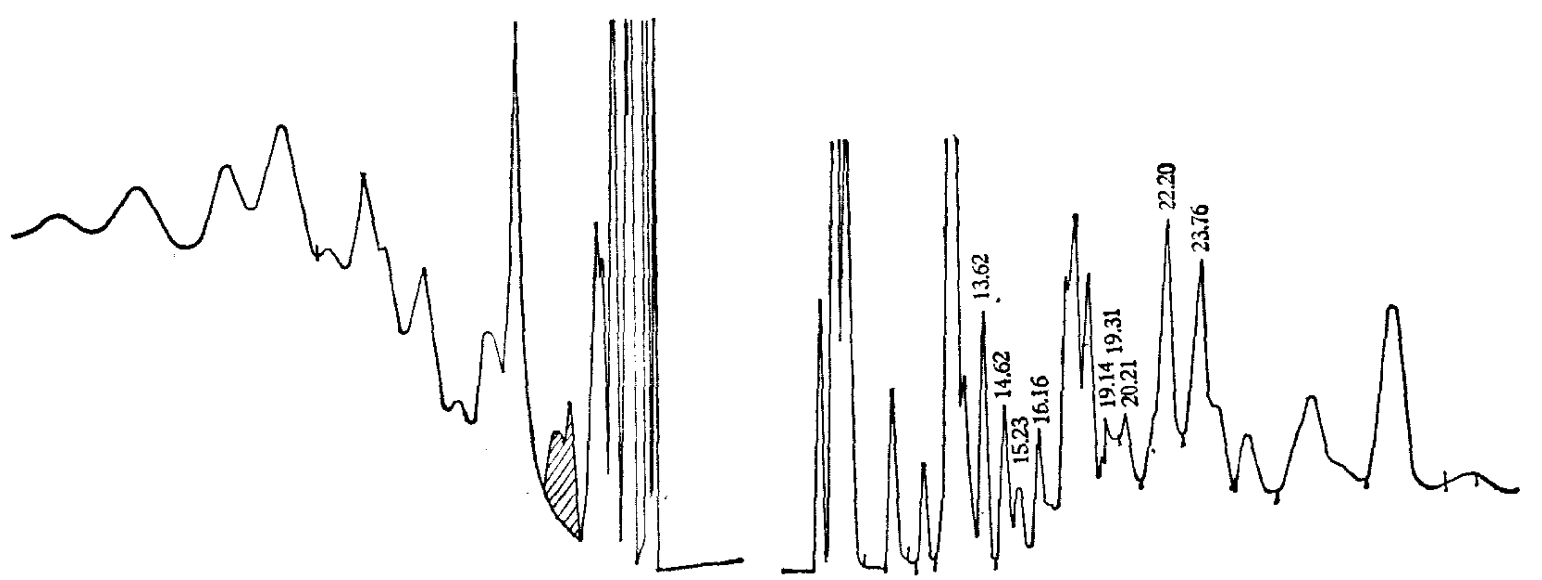

图 1 感染鸡胚卵黄藵

图 2 派氏立克次体 FS110 株

而且还能够区分 5 种不同感染材料. 正常对照材料 17 分钟处的色谱峰和 19,20 分钟处的峰 合并为复合峰，而其他感染材料 17 分钟处则出现单独色谱峰. 5种感染材料的 18 (左)、19 (中)和 20 分钟处都出现一个三重峰: 小株 Kaplan 株的这组峰中间峰最高; 蒙塔那 B 株的 
左、中两峰近似等高; 派氏 FS110 株(图 3)、立氏 R 株和精河株的左、中、右三峰的高度逐次降 低. 此外,立氏 $\mathrm{R}$ 株 30 分钟处色谱峰明显高于 32 分钟处峰. 精河和派氏二者很相似, 但后者 的 15 和 17 分钟两峰近似等高, 而前者 15 分钟处峰略高于 17 分钟处峰. 这一结果和第二组 的结果基本相似.

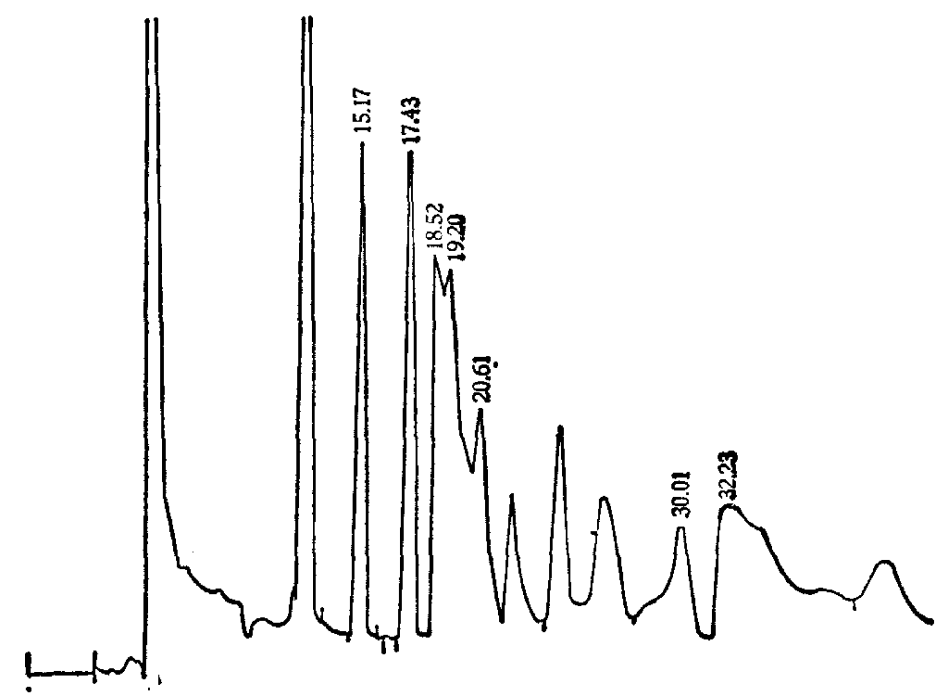

图 3 派氏立克次体 FS110 株

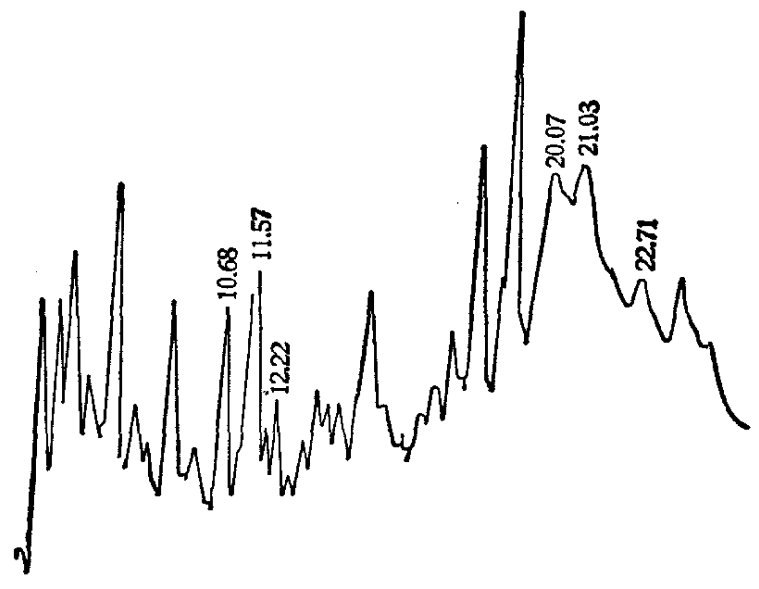

图 4 派氏立克次体 FS110 株

（4）第四组条件绘制的图谱也各具特 征. 例如正常对照 11.58 分钟处出现特高的 单峰, 22.70 分钟处却无峰; 感染材料在此两 个位置上都有色谱峰，但 11.50 分钟处是低 矮峰. 精河株 12.18 分钟处峰高相当于 11.53 分钟处的 $\frac{2}{3}, 20.84$ 和 21.05 分钟处又有明显 双峰。派氏 FS110 株(图 4)和立氏 R 秼 11.50 分钟处峰高不低于 10 分钟处峰, 但派氏的 20.07 和 21.03 分钟处的双峰峰高相当于左侧 峰的 $\frac{1}{2}$, 而立氏的相应值却为 $\frac{1}{4}$. 小株 Kaplan 株和蒙塔那 B 株 11.50 分钟处峰低于左侧峰, 但小株 12.21 分钟处峰高相当于 11.55 处的 $\frac{2}{3}$, 而 蒙塔那 B 株相应比值则只有 $\frac{1}{2}$.

\section{四、讨论}

由上述结果得知，第一、二组色谱条件只能区分正常卵黄襄和感染立克次体材料，而 5 种 立克次体感染材料则难以区分.

第三组条件所得结果比较理想,它不但能显示出正常和感染材料的显著差开,而且还能区 分 5 种立克次体感染材料. 这和我们用这组条件对某些需氧牙狍杆菌的鉴定结果相似 ${ }^{[4]}$. 这 
说明氮磷鉴定器在鉴别生物材料 (尤其是含有大量蛋白质成分的材料) 具有独特的优点.

第四组条件得到的信息量 (峰的数目) 最多, 结果和条件三相似. 说明氢焰电离鉴定器在 微生物分类鉴定工作中是一种被广泛采用的通用鉴定器.

本实验结果和昌正文等人用免疫学和血清学方法对上述 5 种斑点热群立克次体鉴定结果 很相近 ${ }^{[5]}$. 例如精河株和派氏 FS110 株的血清学结果是相近的.

用裂解气相色谱法直接对立克次体感染的鸡胚材料进行鉴别分析可以节省大量实验动物 和血清学试剂, 尤其可以大大缩短检验时间. 初步的实验结果看到了这种方法的可行性, 但要 做为一种鉴别诊断方法仍有必要进一步研究。

致谢: 本文承谢少文教授、汪民副教授审阅; 实验过程中得到本院仪器中心室盛世淑副主任和甘阳生、郑 文奎等大力协助, 谨致深切的谢意.

\section{参考文献}

[1] 周 方,微生物学通报, 7(1980), 3: 136 .

[2] 周 方、于盆华、古惠英、谭立,中华微生物和免疫学杂志, 2(1982)，1: 16 .

[3] 周 方、吴爱棣、周小宁,中华结核和呼吸系疾病杂志，6(1983)，2: 65 .

[4]周 方、刘聿太、王大暳, 微生物学报 (待发婊).

[5] 吕正文、努照满、汪 民、张远富、孙东莲, 中华微生物和免疫学杂志, 2(1982)，1: 36 . 